\title{
Synthesis of Cubic Phase-Co Microspheres by Mechanical Solid-State Reaction-Thermal Decomposition and Research on Its Growth Kinetics
}

\author{
Ying Deng, ${ }^{1,2}$ Yanhua Zhang, ${ }^{1}$ Lingling Peng, ${ }^{1}$ Xiaolong Jing, ${ }^{1}$ and Hui Chen ${ }^{1}$ \\ ${ }^{1}$ Research Institute for New Materials Technology, Chongqing University of Arts and Sciences, Chongqing 402160, China \\ ${ }^{2}$ State Key Laboratory of Powder Metallurgy, Central South University, Changsha 410083, China
}

Correspondence should be addressed to Ying Deng; dengying.163@163.com and Yanhua Zhang; zyhcoco@163.com

Received 12 July 2016; Revised 26 August 2016; Accepted 19 September 2016

Academic Editor: Amit Bandyopadhyay

Copyright (C) 2016 Ying Deng et al. This is an open access article distributed under the Creative Commons Attribution License, which permits unrestricted use, distribution, and reproduction in any medium, provided the original work is properly cited.

\begin{abstract}
Cubic phase cobalt (Co), which can be used as a key component for composite materials given its excellent ductility and internal structure, is not easy to obtain at room temperature. In this study, oxalic acid and cobalt nitrate are used as raw materials to synthesize the cobalt oxalate precursor, which has a stable structure with a five-membered chelate ring. Cobalt oxalate microspheres, having a high internal energy content, were prepared by using mechanical solid-state reaction in the presence of a surfactant, which can produce spherical micelles. The thermal decomposition of the precursor was carried out by maintaining it in a nitrogen atmosphere at $450^{\circ} \mathrm{C}$ for $3 \mathrm{~h}$. At the end of the procedure, $100 \mathrm{~nm}$ cubic phase-Co microspheres, stable at room temperature, were obtained. Isothermal and nonisothermal kinetic mechanisms of cobalt grain growth were investigated. The cubic-Co grain growth activation energy, $Q$, was calculated in this study to be $71.47 \mathrm{~kJ} / \mathrm{mol}$. The required reaction temperature was low, making the production process simple and suitable for industrial applications.
\end{abstract}

\section{Introduction}

Composite materials, such as cermet and cemented carbide, which are composed of a metal hard phase and binder phase, exhibit an excellent performance and are the best choice for applications in the manufacturing and military industries [1]. On the other hand, their application is limited due to the low toughness of the hard phase [2]. Cobalt (Co) powder, which has better ductility than other metals, is being widely used as the binder phase and is a key to improve the strength and toughness of the alloy materials [3]. A study of its production process and properties therefore has an important scientific theoretical value.

At room temperature, Co generally possesses a mixed structure, containing both a cubic structure (face-centered, fcc) and a hexagonal structure (close-pack, hcp) [4]. CubicCo crystals have four groups of slip systems and 12 slip planes, while the hcp-Co structure has only one slip system and three slip planes, as shown in Figure 1. The plasticity of hcp-Co is lower than that of cubic-Co, given the lower number of independent slip planes of the former $[5,6]$.
Furthermore, cubic-Co easily forms an annealing twin in the cooling processes $[7,8]$, which has coincidence site lattice (CSL) to effectively block cracks and improve the ductility of the material $[9,10]$. Therefore, cubic-Co has better ductility and plays an important role in improving the toughness of composite materials.

Additionally, previous studies have shown that a spherical morphology of the powder leads to a small contact surface and can thus enhance dispersion of mixed powders in composite materials $[11,12]$ and improve the mechanical, physical, and chemical properties of micro/nanostructured materials compared to coarse-grained materials [12]. The use of microsphere powders is an optimal choice for the preparation of high performance composite materials [13].

To prepare cubic-Co, the first requirement is that of making the particles reserve enough internal energy to keep stable at room temperature. Currently, many preparation methods for micro/nanostructured powders have been reported, such as the precipitation method, solid reaction method, hydrothermal method, sol-gel method, vapor deposition method, and thermal decomposition method [14-16]. If the 


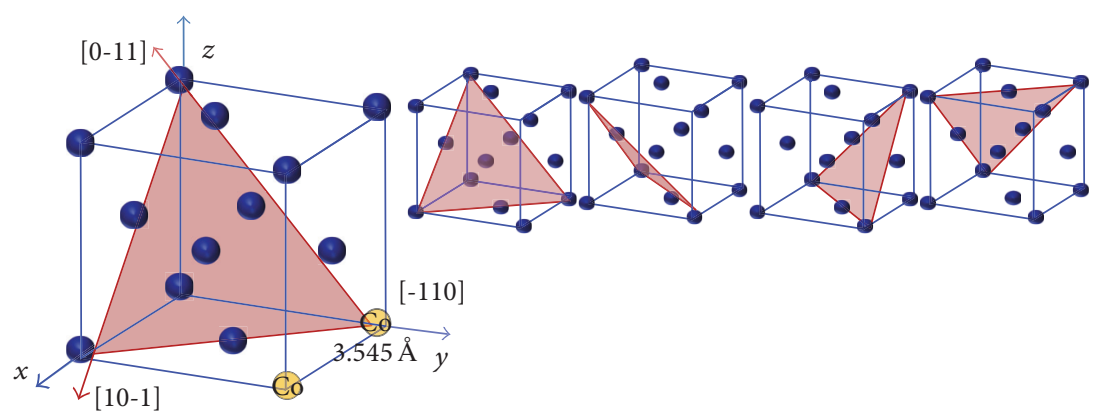

(a)

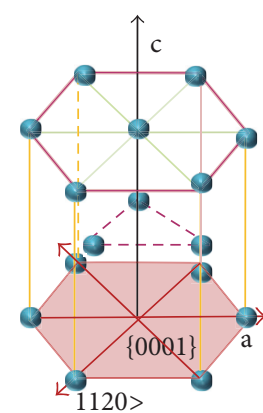

(b)

FIGURE 1: Crystalline structures and the slip system of cobalt: (a) cubic-Co and (b) hcp-Co.

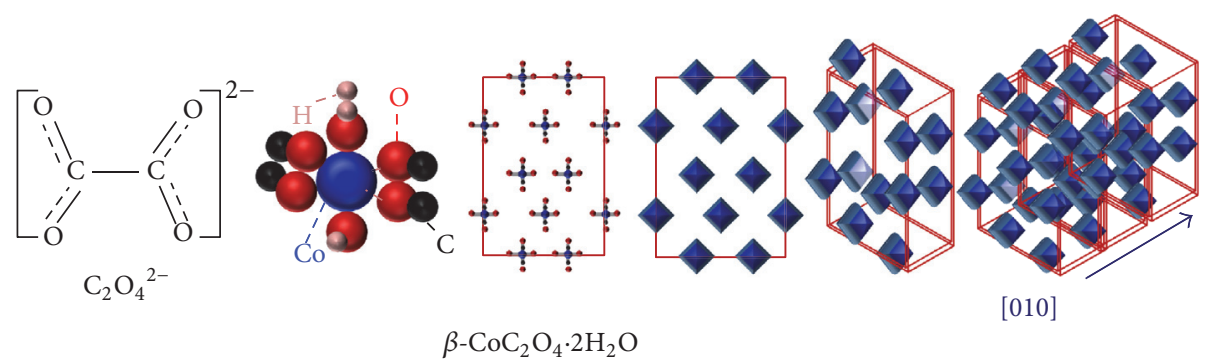

FIGURE 2: Crystal structure of cobalt oxalate.

features of the chemical and physical preparation methods are combined, the particle size is easy to control and the internal energy of particles can be reserved. According to this observation, in this paper, a mechanical (high energy) solid-state reaction method has been used to prepare a cobalt oxalate precursor and obtain cubic-Co microspheres by thermal decomposition. The cobalt grain energy storage, inhibition mechanism of the phase transition, and grain growth kinetics in the temperature range $350^{\circ} \mathrm{C}-750^{\circ} \mathrm{C}$ were also studied.

\section{Preparation Strategy and Experimental Procedure}

2.1. Preparation Strategy. From the perspective of crystal stability, a precursor having a stable structure is necessary to obtain stable Co. Oxalic acid possesses a double tooth structure, which is formed by a $\pi$ system plane (eight electrons) and is the most stable type of chelate [17] (shown in Figure 2). Cobalt oxalate is therefore suitable to be used as precursor to obtain stable Co. Since Co will inherit its morphology from its precursor [4], the experimental strategy consists in firstly preparing cobalt oxalate. Cobalt oxalate grows as a rod-like crystal (crystal structure and growth mechanism are shown in Figure 2) and, on this basis, we have developed a strategy to fabricate a spherical precursor through solid-state reaction (mechanical ball milling), which envisages the use of surfactant cetyltrimethyl ammonium bromide (CTAB) spherical micelles. Suitable concentration of CTAB can efficiently prevent particle from growing up and agglomeration. The effect of CTAB will be little when the concentration of CTAB is lower than 10 times of critical concentration. But if the adding amount of CTAB is excessive, it cannot generate spherical micelles $[18,19]$. According to our previous experiments of CTAB adding, $2-3 \mathrm{wt} \%$ is the optimal concentration of CTAB, so we choose 2 wt $\%$ CTAB to be added in this experiment. Using this strategy, the size, morphology, and structure of the precursor can be easily controlled, and the energy reserve may be determined by choosing the adequate operating conditions during the mechanical ball milling. Finally, the precursor was decomposed and, by controlling the heat retaining time and heating temperature, the target cubic-Co powder was obtained. The schematic of the research strategy of this paper is shown in Figure 3.

\subsection{Experimental Procedures}

Cobalt Precursor Samples. $\mathrm{CoC}_{2} \mathrm{O}_{4} \cdot 2 \mathrm{H}_{2} \mathrm{O}$ and $\mathrm{Co}\left(\mathrm{NO}_{3}\right)_{2}$. $6 \mathrm{H}_{2} \mathrm{O}$, along with $2 \mathrm{wt} \%$ surfactant CTAB, based on the total mass of reagents, were prepared in quantities in accordance with the stoichiometry of the reaction (formula (1)). The reagents were thoroughly mixed and divided into two samples: sample (b) was transferred to a mortar and grinded until the reaction was complete (became pink); sample (a) was placed into a $100 \mathrm{~mL}$ agate grinding cylinder for ball milling (planetary ball mill, with agate ball/powder feed ratio of $2: 1$, milling speed $300 \mathrm{r} / \mathrm{min}$, and milling time $3 \mathrm{~h}$ ). After that, the two samples were filtrated and washed separately. Alcohol was then added to prepare precursor solutions of samples (a) and (b). The obtained solution was spray dried 


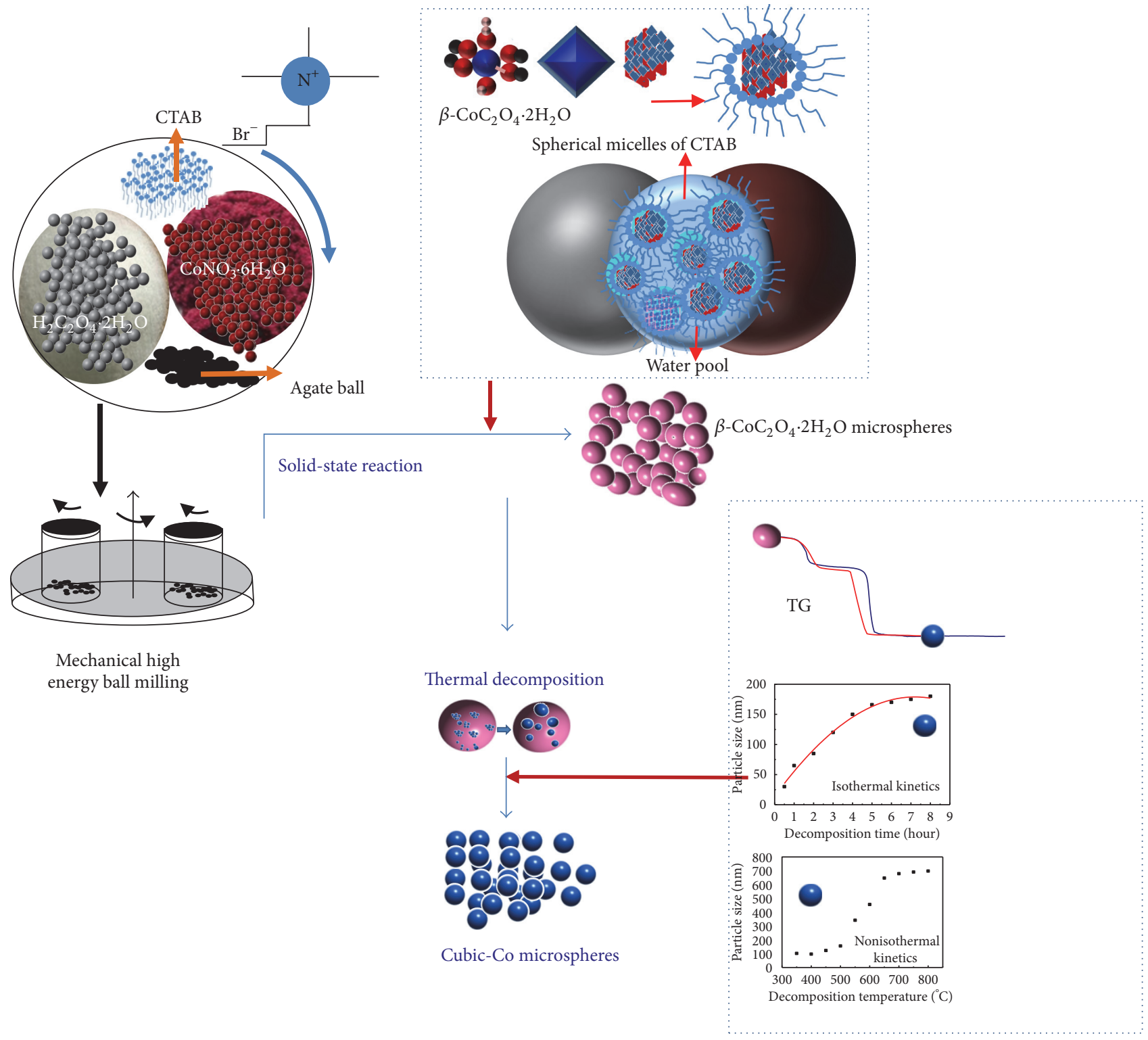

FIGURE 3: Schematic of the research strategy.

and the uniformly dispersed cobalt precursors samples were obtained.

Cobalt Samples. The two precursors sample powders were decomposed under a nitrogen atmosphere. The decomposition temperature was in the range $350^{\circ} \mathrm{C}-750^{\circ} \mathrm{C}$, and the time required was between 0.5 and $8 \mathrm{~h}$. Samples were then cooled to room temperature under a nitrogen atmosphere and the target Co powder samples (a) and (b) were obtained.

All the chemical reagents used in this study were of analytical grade and used as received without further purification.

$X$-Ray Diffraction (XRD) Study. The X-ray diffraction study $\left(\mathrm{CuK} \alpha, \lambda=0.154 \mathrm{~nm}\right.$, step $\left.=0.02^{\circ} / \mathrm{s}\right)$ was carried out using the XRD equipment model D/MAX2500VL/PC. The full width at half maximum (FWHM) was calculated by using Jade software after refining diffraction peaks. Grain size was estimated using Scherrer formula.

Morphology and Microstructure Study. The particle morphology and microstructure were studied on a JEOL6490LV (Japan) field emission scanning electron microscope (SEM) and a JEOL-2100F (Japan) field emission transmission electron microscope. The HORIBA-LA-950A2 laser (Japan) was used to determine the particle size distribution.

Thermo Gravimetric (TG) Study. An STA-409PC thermal analyzer (Germany) was used to carry out the TG thermal 


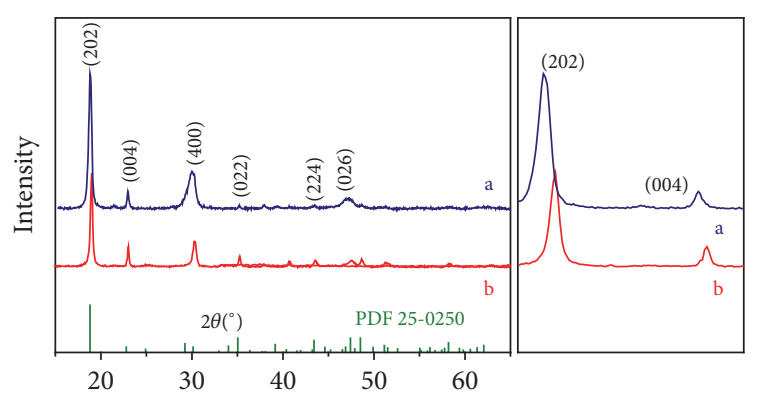

FIGURE 4: XRD images of the precursor (cobalt oxalate): (a) mechanical solid-state reaction; (b) hand-grinding.

study (nitrogen atmosphere, gas flow rate of $30 \mathrm{~mL} / \mathrm{min}$, and heating rate of $10^{\circ} \mathrm{C} / \mathrm{min}$ ).

\section{Results and Discussion}

3.1. Structure and Morphology of Precursor. In this experiment, the proposed equation for the solid-state reaction process is as follows:

$$
\begin{gathered}
\mathrm{H}_{2} \mathrm{C}_{2} \mathrm{O}_{4} \cdot 2 \mathrm{H}_{2} \mathrm{O}+\mathrm{Co}\left(\mathrm{NO}_{3}\right)_{2} \cdot 6 \mathrm{H}_{2} \mathrm{O} \longrightarrow \\
\mathrm{CoC}_{2} \mathrm{O}_{4} \cdot 2 \mathrm{H}_{2} \mathrm{O}+2 \mathrm{HNO}_{3}+6 \mathrm{H}_{2} \mathrm{O} .
\end{gathered}
$$

Samples (a) and (b) are employed, respectively, for the mechanical solid-state reaction and as hand-grinding solidstate reaction product. Figure 4 shows the XRD patterns for samples (a) and (b). The figure shows that characteristic peaks of all samples coincide with PDF25-0250, confirming that the solid-state reaction (1), which includes both the hand-grinding and mechanical solid-state reactions, occurs and that the product is orthorhombic $\mathrm{CoC}_{2} \mathrm{O}_{4} \cdot 2 \mathrm{H}_{2} \mathrm{O}$. It is apparent that the peak of sample (a) significantly shifts to low angles, and the peak width is larger. This indicates that the mechanical force of the mechanical ball milling makes the crystal reserve a certain amount of internal energy allowing the cobalt oxalate lattice to expand and causing grain refinement. Figures 5(a) and 5(b) are cobalt oxalate SEM images for samples (a) and (b), respectively. As can be seen from the figures, sample (b) has a rod-like shape, with an aspect ratio greater than 3. Sample (a) is obviously refined, with a spherical morphology and a particle size of about $100 \mathrm{~nm}$. This indicates that the nucleation and aggregation of the mechanical solid-state reaction product can be effectively controlled and that the addition of 2 wt. $\%$ of CTAB makes the morphology of the product spherical.

The rate with which the mechanical solid-state reaction proceeds depends on the nucleation and diffusion processes [19]. In this experiment, ball milling and the exothermic reaction provide the thermal energy needed by solid reactant diffusion and product nucleation. The two reactants of formula (1), containing large amounts of crystal water that forms numerous tiny pools, go through the initial mechanical solidstate reaction, and as the CTAB generates spherical micelles in the pools, the reactants are dissolved and continue to react in the micelles. The product immediately precipitates, and the micelles limit nucleation and prevent further growth in the dominant growth direction as well as agglomeration, leading to the reaction of nanosized spherical particles.

Cubic-Co has a higher free energy content than hcp-Co and a martensitic transformation will occur during cooling [4], In the mechanical ball milling process, lattice defects of powders will increase, and according the some reports $[4,20]$, the extra energy $\Delta G^{*}$ caused by lattice defects and grain refinement can be expressed according to formula (2), wherein $\mu$ is the shear modulus of the powder, $b$ is Burgers vector, $\beta$ is XRD diffraction peak half-width caused by lattice distortion:

$$
\Delta G^{*}=\frac{\mu m \beta^{2}}{4.35 \rho}+3 \gamma \frac{m}{\rho \Delta r}+\frac{2 K m \gamma}{r \rho} .
$$

In this experiment, the calculated result for $\Delta G^{*}$ (after $3 \mathrm{~h}$ milling) according to formula (2) is greater than $1 \mathrm{KJ} / \mathrm{mol}$, which is enough for the direct generation of single-cubic phase Co in cobalt oxalate during the thermal decomposition reaction (1) and to overcome the phase transformation during cooling, thus allowing it to maintain the structure even at room temperature (cubic-Co free energy of formation is $251 \mathrm{~J} / \mathrm{mol}$ and the critical driving force of allotropic transformation is $-160 \mathrm{~J} / \mathrm{mol}$ ) [4].

Figure 6 shows TEM and high-resolution diffraction images for the cobalt oxalate sample (a). The image shows that the crystal contains a large number of dislocations and slips in it, which distorts the lattice and increases the defect density. The images give important information regarding the result of chemical reactions under mechanical action and prove that the cobalt oxalate powder produced in this experiment has a high energy content, which is sufficient for the preparation of cubic-Co. This is also evidenced by the XRD patterns in Figure 4.

3.2. Cubic-Co Grains Growth Kinetics. Nanoparticles of cobalt oxalate prepared in this experiment have a high energy. The growth law of the Co particles by thermal decomposition is quite different from that of ordinary particles. Thermal power is the key parameter that determines the grain size and, in the decomposition process, the heat retaining time and decomposition temperature determine the level of thermal power. To ensure that nano-cubic-Co powder can be obtained it is necessary to study the growth kinetics of thermal decomposition.

3.2.1. Grain Growth Law under Isothermal Conditions. The possible reaction of precursors undergoing thermal decomposition in a nitrogen atmosphere is as follows:

$$
\mathrm{CoC}_{2} \mathrm{O}_{4} \cdot 2 \mathrm{H}_{2} \mathrm{O} \longrightarrow \mathrm{Co}+2 \mathrm{CO}_{2}+2 \mathrm{H}_{2} \mathrm{O} \text {. }
$$

Figure 7 shows the results of the TG analysis samples (a) and (b), respectively. According to Figure 7, it can be understood that the residual mass after thermal decomposition for the two cobalt oxalate samples was approximately $32 \%$, indicating that reaction (3) was carried out. It can be easily observed from Figure 7 that the decomposition 


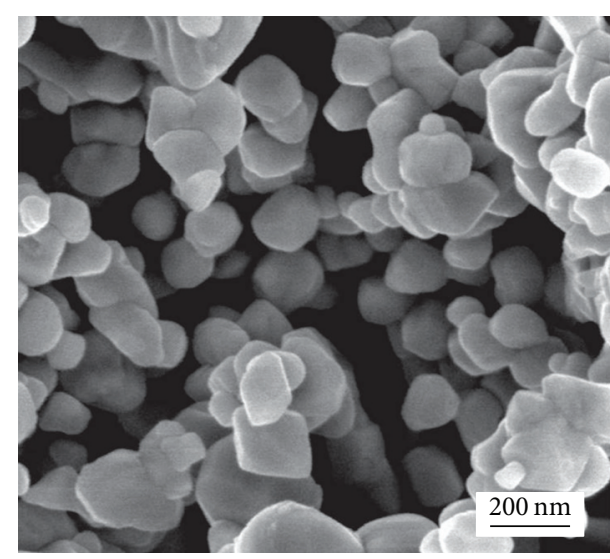

(a)

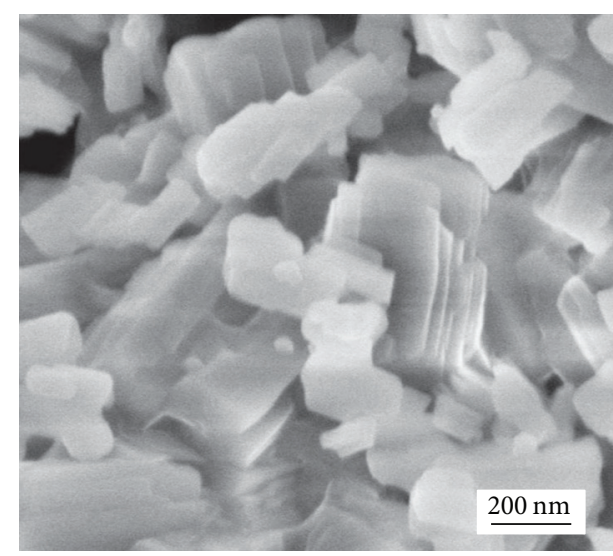

(b)

FIGURE 5: SEM images of the precursor: (a) mechanical solid-state reaction; (b) hand-grinding.

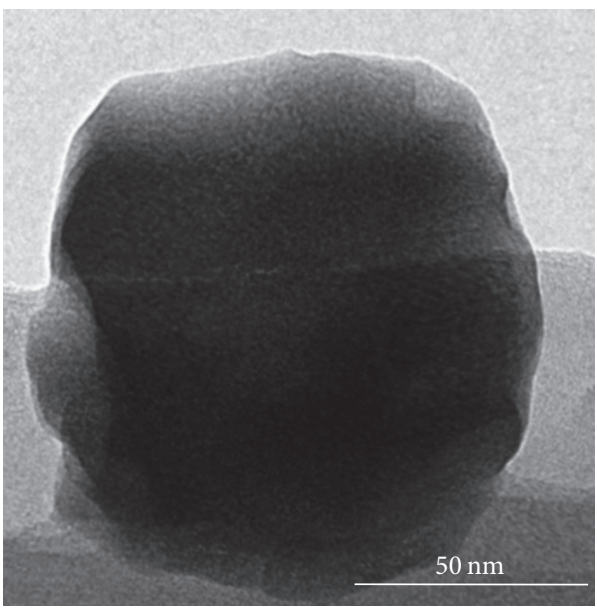

(a)

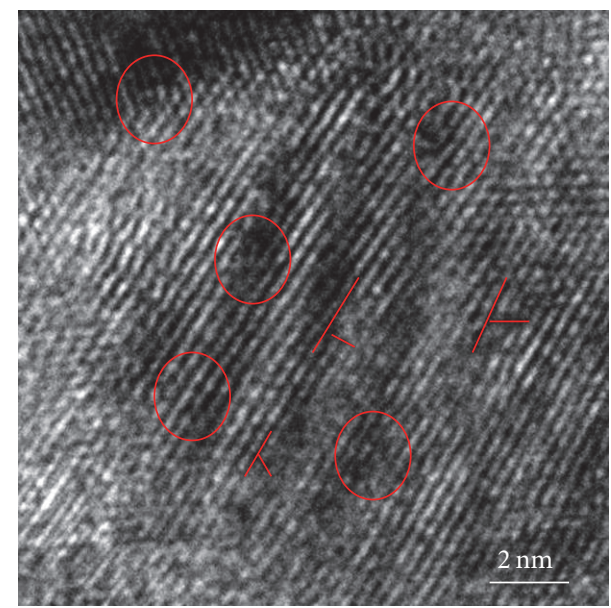

(b)

FIGURE 6: TEM and high-resolution images for $\beta-\mathrm{CoC}_{2} \mathrm{O}_{4} \cdot 2 \mathrm{H}_{2} \mathrm{O}$ sample (a) (mechanical solid-state reaction).

temperature for sample (a) was lower than that of the handgrinding sample (b), indicating that the precursor produced by mechanical ball milling has a high activity and is easy to decompose. Figure 7 (a) shows that at about $328.8^{\circ} \mathrm{C}$ the precursor decomposition begins and a phase transition gradually occurs. When the temperature rises to about $410.5^{\circ} \mathrm{C}$ there is almost no loss of quality in the reaction, suggesting that the precursor decomposition temperature should be higher than $410.5^{\circ} \mathrm{C}$. Therefore, the isothermal decomposition condition of this experiment is set to $450^{\circ} \mathrm{C}$, which is slightly higher than the decomposition temperature of the precursor, and enables the precursor to be completely decomposed with low energy consumption.

Figures 8 and 9 show, respectively, SEM morphology and XRD diffraction patterns for Co powders prepared by using sample (a) of cobalt oxalate after decomposition at $450^{\circ} \mathrm{C}$. The heat retaining times used were $0.5,1,2,3,4$, $5,6,7$, and $8 \mathrm{~h}$. Figure 8 shows that, for retaining times of 0.5-2 h, the Co powder consisted of an agglomeration of very small flocculent particles and that with the increase of the retaining time, the Co powder particle size increased. When the retaining time was increased to $3 \mathrm{~h}$, the Co powder morphology gradually changed to spherical particles. After the time increased to 5 hours, particles began to adhere, and the grain no longer grew significantly. As can be seen from Figure 9, referring to samples decomposed at $450^{\circ} \mathrm{C}$, each peak is present at values corresponding to cubic-Co, according to Powder Diffraction File (PDF) of cubic cobalt (PDF894307). This proves that the reserve energy, $\Delta G^{*}$, of this experiment was sufficient to make the cobalt precursor produce single-phase cubic-Co in the thermal decomposition reaction and prevent the martensitic transformation, keeping the fcc structure even at room temperature.

The rate of crystal growth can be studied with the German and Munir (GM model) kinetics model [21]:

$$
D^{n}-D_{0}{ }^{n}=\frac{t}{T} \beta e^{-\mathrm{Q} / R T},
$$




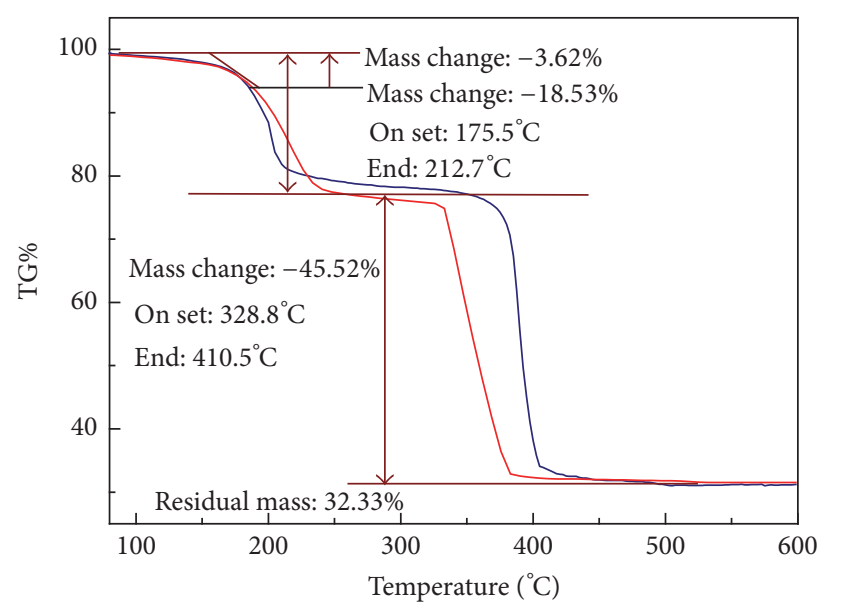

$-\mathrm{a}$

FIGURE 7: TG results for precursor under $\mathrm{N}_{2}$ atmosphere: (a) sample (a) mechanical solid-state reaction; (b) sample (b) hand-grinding.

where $t$ and $T$ are, respectively, the heat retaining time and the absolute temperature, $D_{0}$ is the initial grain size of Co, $D$ is the grain size at each heating time, $n$ is the time index, $Q$ is the activation energy for grain growth, $R$ is the gas constant, and $\beta$ is a characteristic constant of the powder. Assuming that $D_{0}$ is sufficiently small, (4) can be rewritten as

$$
D^{n}=\frac{t}{T} \beta e^{-Q / R T},
$$

which, in logarithmic form, yields

$$
\begin{aligned}
\ln D & =\frac{1}{n} \ln t+\frac{1}{n}\left(\ln \beta-\ln T-\ln \frac{Q}{R T}\right), \\
D & =\frac{k \lambda}{\beta \cos \theta} .
\end{aligned}
$$

On the basis of the results reported in Figure 9, the Scherrer equation (7) was used to calculate the cobalt crystallite size for each period and the results are shown in Figure 10(a). Here, the FWHM of cubic-Co characteristic peaks (111) (200) (200) were used to calculate the crystallite size and to evaluate the averaged value. From (5), the grain growth kinetics $\ln (D)$ versus $\ln (t)$ for the Co powder during the decomposition process at $450^{\circ} \mathrm{C}$ can be obtained. Fitting the experimental points of Figure 10(a), lines having different slopes, corresponding to the two growth stages, were obtained and plotted in Figure 10(b). By evaluating the slopes, it was possible to determine rates of the rapid growth phase, $n_{1}=3.41$, and of the slow growth phase, $n_{2}=5.01$.

As for GM models, $n$ represents the growing ability by breaking the grain boundary resistance. The smaller the value of $n$, the greater the ability of the crystals to grow. When $n>$ 3.5 , the crystal growth is mainly affected by surface diffusion [22]. The mechanism responsible for the growth of cubicCo grains under isothermal decomposition conditions can therefore be explained as follows: during the initial decomposition stage $(0.5-2 \mathrm{~h})$, many critical nuclei are formed in the precursor particles, and the surface energy drives grains to agglomerate, forming larger grains; with the increase of holding time $(>3 \mathrm{~h})$, the grains become increasingly large, and the surface energy and crystal structure defects are both reduced, slowing down the rate of grain growth and forming new grain boundaries. The final lattice tends to be stabilized, forming stable grains. To sum up, we can conclude that the law of cubic-Co growth kinetics is determined by its surfactant and internal energy.

\subsubsection{Grain Growth Law under Nonisothermal Conditions.} Isothermal kinetic studies indicate that the retaining time for mechanical solid-phase reaction sample (a) should be longer than $3 \mathrm{~h}$. Moreover, Figure 10 shows that Co particles of sample (a) were fully developed when the holding time was $3 \mathrm{~h}$, and XRD results show a sharp peak in correspondence of this time, which proves that a large yield of particles having the appropriate grain size was obtained. Therefore, the retaining time of $3 \mathrm{~h}$ was identified as the most suitable for thermal decomposition as per the conditions of this experiment.

Nine different temperature values were selected to study the decomposition occurring with a heat retaining time of $3 \mathrm{~h}$ to obtain cubic-Co powders. The average particle size was measured and results are shown in Figure 11(a). From this figure, it is evident that the particle size rapidly grew when the temperature was greater than $550^{\circ} \mathrm{C}$ (critical temperature) [23]. At $450^{\circ} \mathrm{C}$, the grain growth was not obvious, which proves that at this temperature Co was completely decomposed.

The grain growth law under nonisothermal conditions can be studied through the activation energy and can be estimated from the average grain size and the thermal decomposition temperature, using the Arrhenius formula [24]:

$$
D^{n}-D_{0}^{n}=k_{0} t e^{-\mathrm{Q} / R T}
$$

In (8), $D_{0}$ is the initial size of the crystal grains of Co, $D$ is the grain size at the heating time $t, K_{0}$ is a constant, $n$ is a time index, $Q$ is the activation energy for grain growth, $R$ is the gas constant, $T$ is decomposing reduction temperature, $R$ is the ideal gas constant $(R=8.314 \mathrm{~J} / \mathrm{mol} / \mathrm{K})$. If $D_{0}=0$, (8) becomes

$$
n \ln D=A-\frac{Q}{R T} \quad\left(A=\ln \left(k_{0} t\right)\right) .
$$

According to Figure $11(\mathrm{a})\left(400^{\circ} \mathrm{C}-700^{\circ} \mathrm{C}\right)$, a linear regression to fit $\ln (D)$ and $1 / T$ was carried out and the results are shown in Figure 11(b). Equation (9) shows that a linear relationship existed between $\ln (D)$ and $T^{-1}$ and the slope was $-Q / R$. The fitting result was substituted into (9) as shown below:

$$
n \ln D=10.234-\frac{3.439}{T}
$$




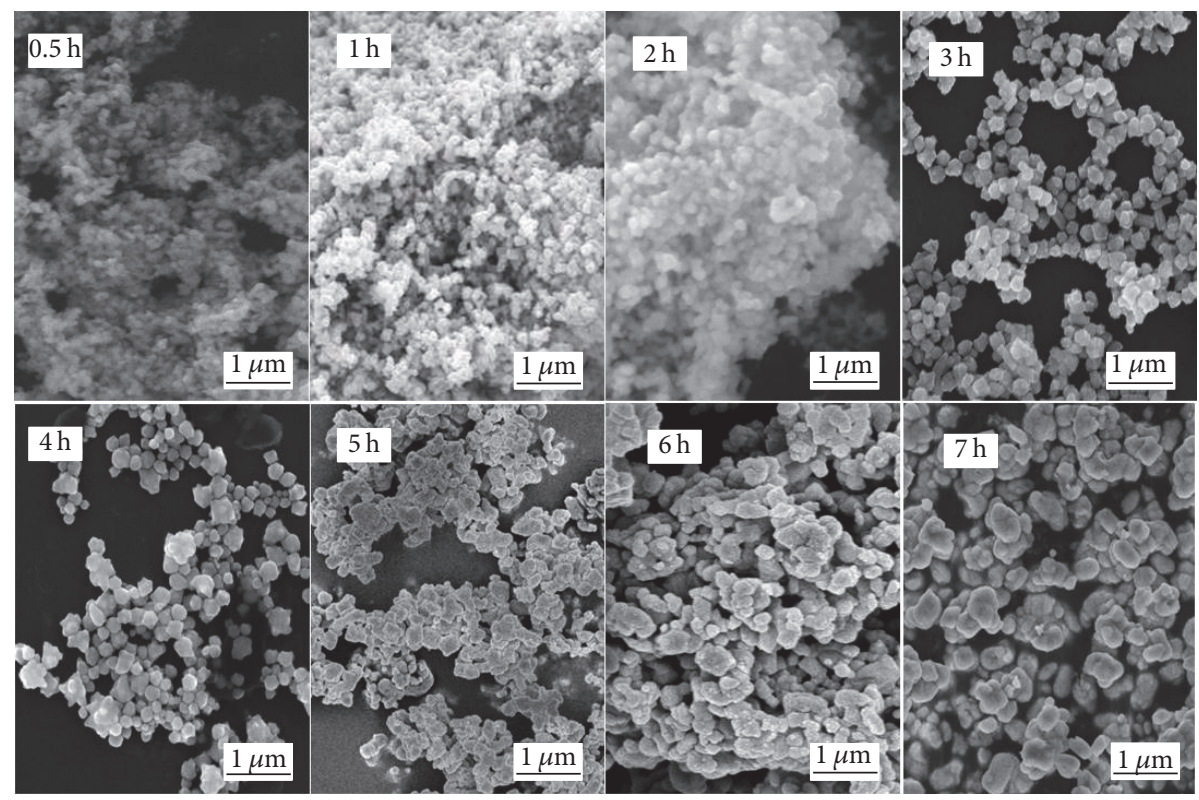

Figure 8: Co particle size of sample (a) changes with different heat retaining times $\left(450^{\circ} \mathrm{C}\right)$.

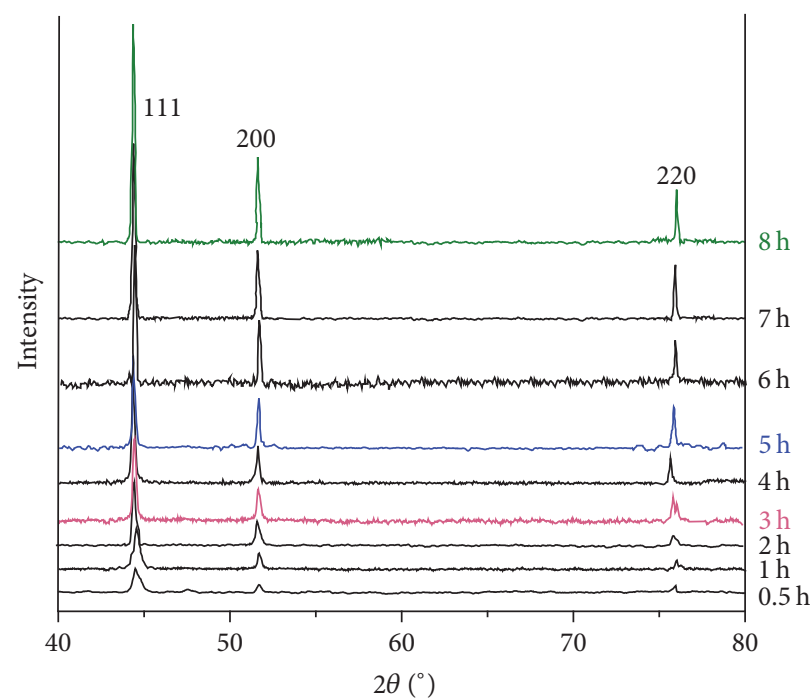

FIGURE 9: XRD images for Co sample (a) with different heat retaining times $\left(450^{\circ} \mathrm{C}\right)$.

Substituting $n_{1}=3.41$ (rapid growth phase) into (9), the Co grain growth activation energy was calculated to be equal to $Q=71.47 \mathrm{~kJ} / \mathrm{mol}$. This value is very small, far less than the grain boundary diffusion activation energy of $115 \mathrm{~kJ} / \mathrm{mol}$ of the large grains of pure Co [25].

The activation energy calculated for this experiment shows that the surface energy and the internal energy of Co particles are large and that the controlling factor in grain growth is not only diffusion, but also the reactivity of the grain surface, which reduces the energy requirement for grain growth. The results reported here prove that the thermal decomposition temperature for precursor sample (a) must have been lower than the one for sample (b), in accordance with the SEM, XRD, and TG test results (Figure 7).
3.3. Thermal Decomposition Condition for Target. To summarize the analyses carried out above, the optimal decomposition conditions to obtain the target Co powder are decomposition time of $3 \mathrm{~h}$ in a nitrogen atmosphere at $450^{\circ} \mathrm{C}$.

Figures 12(a) and 12(b) show the XRD and TEM images relative to Co powders from the cobalt oxalates samples (a) and (b), respectively. From Figure 12 it can be observed that sample (b) was obviously of a mixed crystal type, in agreement with $\Delta G^{*}$ values calculated in this experiment. When the mechanical force is sufficiently large (mechanical ball milling), the extra $\Delta G^{*}$ is enough to lead to the generation of single-phase cubic-Co during the thermal decomposition reaction and to keep the same structure down to room temperature. The TEM image Figure 12(b) shows 


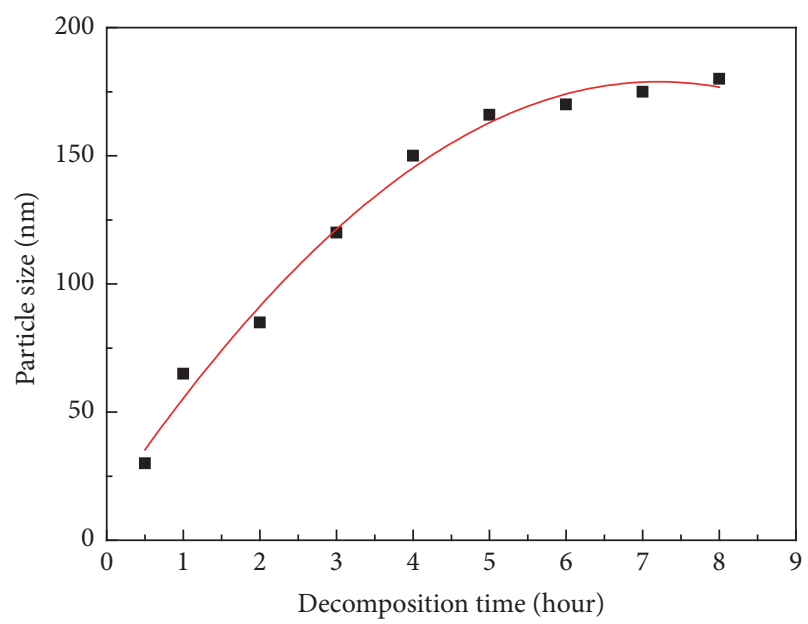

(a)

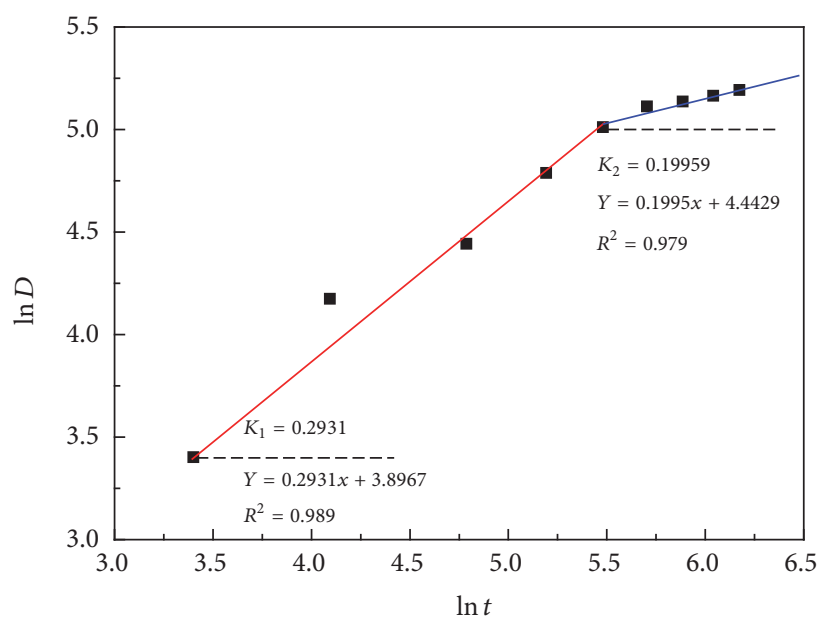

(b)

Figure 10: Different heat retaining times $\left(450^{\circ} \mathrm{C}\right)$ for precursor sample (a) decomposition: (a) particle size for Co; (b) kinetics model for growth of Co.

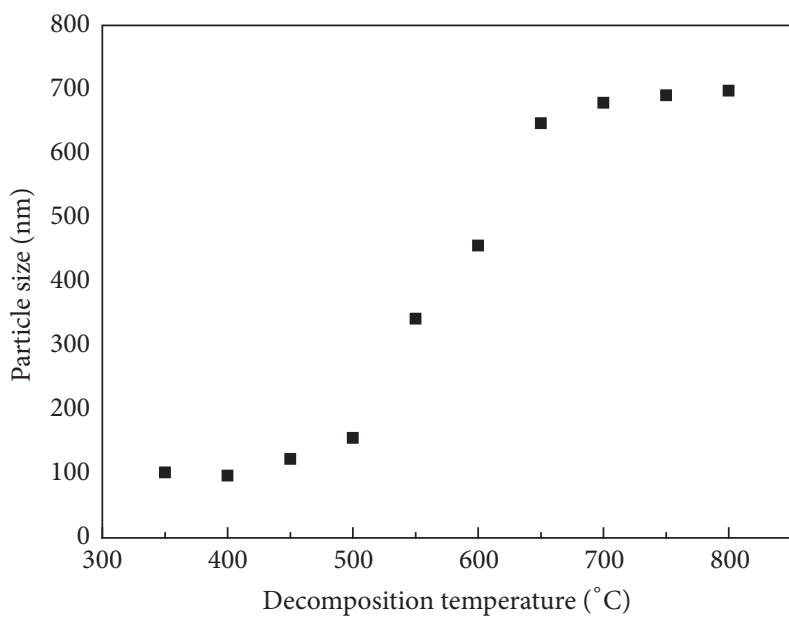

(a)

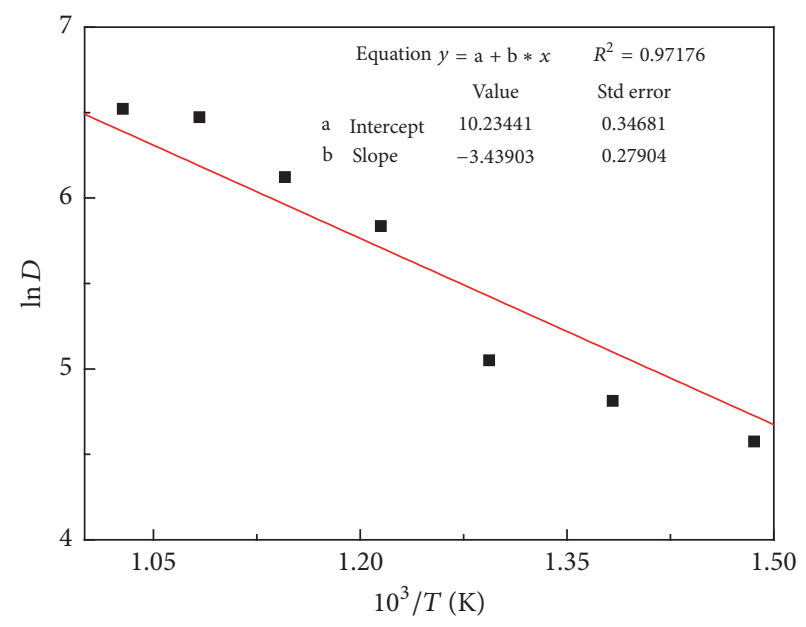

(b)

Figure 11: (a) Average particle size of Co sample (a) as a function of temperature; (b) relationship between $\ln (D)$ and $10^{3} / T$.

that sample (b) presents a dendritic morphology, inheriting the morphology of its cobalt oxalate precursor.

Figure 13 shows the particle size distribution patterns for the target Co powders sample (a). It can be clearly observed from Figure 13 that the Co particles of sample (a) were evenly distributed, well-dispersed, and the average particle size is around $100 \mathrm{~nm}$.

\section{Conclusions}

(1) A mechanical solid-state reaction can be used to control crystal morphology and reserve energy during the preparation of spherical cobalt oxalate precursors having a size of about $100 \mathrm{~nm}$. The extra energy $\Delta G^{*}$ is greater than $1 \mathrm{KJ} / \mathrm{mol}$, enough to allow the direct generation of single-cubic phase Co from the thermal decomposition reaction of the cobalt precursor, and to overcome the phase transformation during cooling.

(2) The activation energy of cobalt grain growth, $Q$, evaluated in this study was $71.47 \mathrm{~kJ} / \mathrm{mol}$, which is far lower than the pure Co grain boundary diffusion activation energy. This proves that the activity of the powder produced by mechanical solid-state reaction in this experiment was high, and the activation energy required for grain growth was very small. Spherical cubic phase (fcc) Co microspheres around $100 \mathrm{~nm}$ in size can be prepared by decomposing the precursor at $450^{\circ} \mathrm{C}$ for $3 \mathrm{~h}$ under a nitrogen atmosphere. 


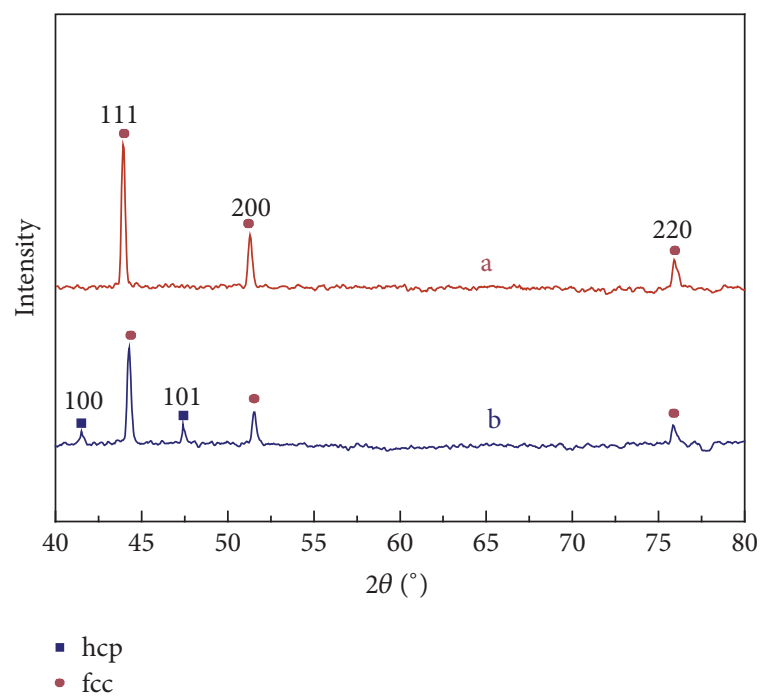

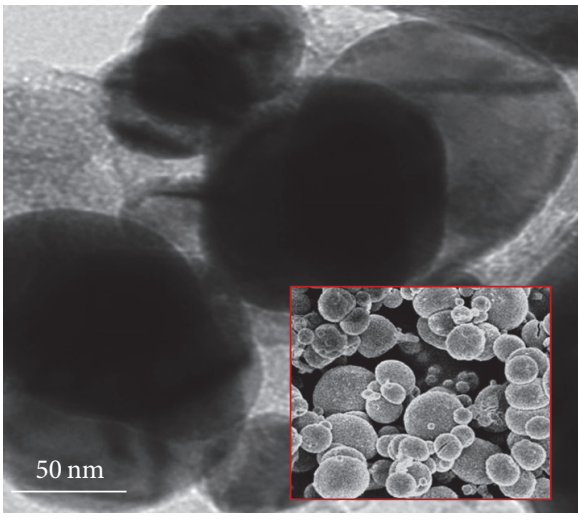

(a)

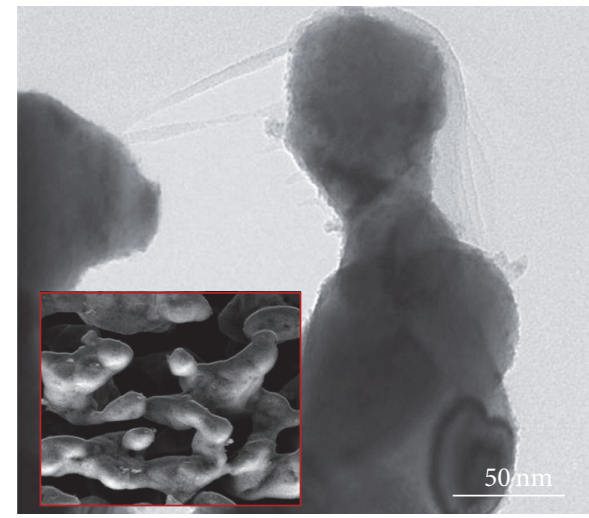

(b)

FIGURE 12: XRD and TEM images for Co (decomposed at 450 $\mathrm{C}$ ): (a) sample (a) mechanical solid-state reaction; (b) sample (b) hand-grinding.

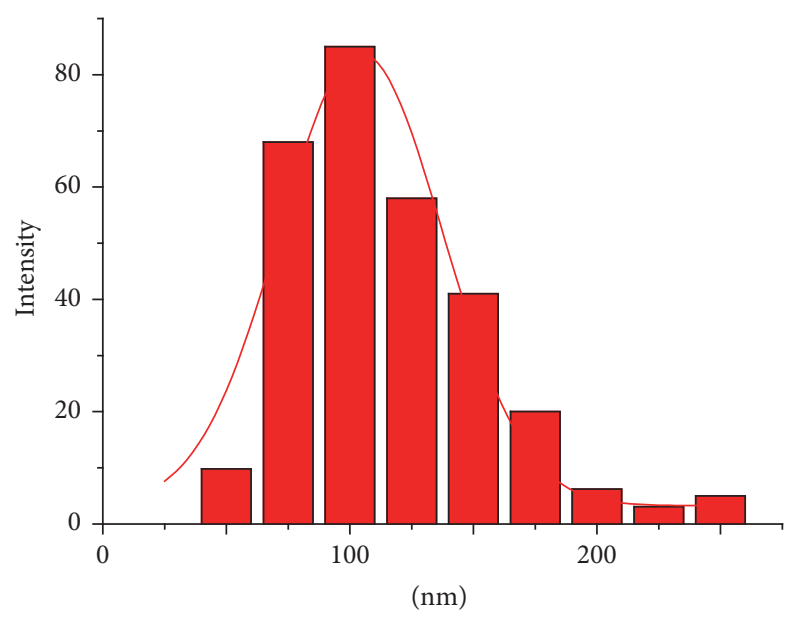

FIGURE 13: Particle size distribution of target Co powders sample (a).

\section{Competing Interests}

The authors declare that there is no conflict of interests regarding the publication of this article.

\section{Acknowledgments}

This work was supported by the scientific research project of Chongqing University of Arts and Science, Y2014CJ26, the cooperation project, "Development of High Strength and Toughness Micro/Nanocermet Materials" between Chongqing University of Arts and Science and Chongqing Academy Science and Technology, the National Natural Science Foundation of China (no. 51274248), and the International Scientific and Technological Cooperation Project of China (no. 2013DFA31440).

\section{References}

[1] M. Bl’anda, A. Duszová, T. Csanádi, P. Hvizdoš, F. Lofaj, and J. Dusza, "Indentation fatigue of WC grains in WC-Co composite," Journal of the European Ceramic Society, vol. 34, no. 14, pp. 3407-3412, 2014.

[2] J. Jung and S. Kang, "Effect of ultra-fine powders on the microstructure of $\mathrm{Ti}(\mathrm{CN})-\mathrm{x}-\mathrm{WCNi}$ cermets," Acta Materialia, vol. 52, p. 1379, 2004.

[3] A. Rajabi, M. J. Ghazali, J. Syarif, and A. R. Daud, "Development and application of tool wear: a review of the characterization of 
TiC-based cermets with different binders," Chemical Engineering Journal, vol. 255, pp. 445-452, 2014.

[4] Y. Deng, X. Xiong, J. P. Zou, L. Deng, and M. J. Tu, "Control of morphology and structure for $\beta$-Co nanoparticles from cobalt oxalate and research on its phase-change mechanism," Journal of Alloys and Compounds, vol. 618, pp. 497-503, 2015.

[5] X. B. Zhang and N. Liu, "Microstructure, mechanical properties and thermal shock resistance of nano-TiN modified TiCbased cermets with different binders," International Journal of Refractory Metals and Hard Materials, vol. 26, no. 6, pp. 575$582,2008$.

[6] A. Jain and S. R. Agnew, "Modeling the temperature dependent effect of twinning on the behavior of magnesium alloy AZ31B sheet," Materials Science and Engineering A, vol. 462, no. 1-2, pp. 29-36, 2007.

[7] G. I. Taylor, "Plastic strain in metals," Journal of the Institute of Metals, vol. 62, pp. 307-324, 1938.

[8] V. Baco-Carles, A. Arnal, D. Poquillon, and P. Tailhades, "Correlation between the morphology of cobalt oxalate precursors and the microstructure of metal cobalt powders and compacts," Powder Technology, vol. 185, no. 3, pp. 231-238, 2008.

[9] T. Baudin, A. L. Etter, and R. Penelle, "Annealing twin formation and recrystallization study of cold-drawn copper wires from EBSD measurements," Materials Characterization, vol. 58, no. 10, pp. 947-952, 2007.

[10] C. S. Pande, B. B. Rath, and M. A. Imam, "Effect of annealing twins on Hall-Petch relation in polycrystalline materials," Materials Science and Engineering: A, vol. 367, no. 1-2, pp. 171175, 2004.

[11] C. V. Kopezky, V. Y. Novikov, L. K. Fionova, and N. A. Bolshakova, "Investigation of annealing twins in F.C.C. metals," Acta Metallurgica, vol. 33, no. 5, pp. 873-879, 1985.

[12] Y. Soumare, C. Garcia, T. Maurer et al., "Kinetically controlled synthesis of hexagonally close-packed cobalt nanorods with high magnetic coercivity," Advanced Functional Materials, vol. 19, no. 12, pp. 1971-1977, 2009.

[13] X. Y. Pan, Y. Chen, X. M. Ma, and L. H. Zhu, "Phase transformation of nanocrystalline anatase powders during high energy planetary ball milling," Transactions of Nonferrous Metals Society of China, vol. 13, pp. 271-272, 2003.

[14] B. Varghese, T. C. Hoong, Z. Yanwu et al., " $\mathrm{CO}_{3} \mathrm{O}_{4}$ nanostructures with different morphologies and their field-emission properties," Advanced Functional Materials, vol. 17, no. 12, pp. 1932-1939, 2007.

[15] X. D. Wang, E. Graugnard, J. S. King, Z. L. Wang, and C. J. Summers, "Large-scale fabrication of ordered nanobowl arrays," Nano Letters, vol. 4, no. 11, pp. 2223-2226, 2004.

[16] D. R. Lide, CRC Handbook of Chemistry and Physics, CRC Press/Taylor and Francis, Boca Raton, Fla, USA, 90th edition, 2009.

[17] E. Foos, R. Stroud, A. Berry, A. W. Snow, and J. P. Armistead, "Synthesis of nanocrystalline bismuth in reverse micelles," Journal of the American Chemical Society, vol. 122, no. 29, pp. 7114-7115, 2010.

[18] J. Xiao and Z. Zhao, Principle of Surfactant Application, Chemical Industry Press, Beijing, China, 2003.

[19] S. Milosevie and M. Ristie, "Thermodynamics and kinetics of mechanical: activation of materials," Seience of Sinering, vol. 30, article 29, 1998.

[20] S. B. Ogale, "Dilute doping, defects, and ferromagnetism in metal oxide systems," Advanced Materials, vol. 22, no. 29, pp. 3125-3155, 2010.
[21] K. Wei, F. Li, and C. Da, "Nanocrystalline rare earthmixed oxides Dyl(-x)SrxCoO(3-y): study on the solid state reaction," Acta Chimica Sinica, vol. 58, no. 1, pp. 71-74, 2000.

[22] S. Shukla, S. Seal, R. Vij, and S. Bandyopadhyay, "Reduced activation energy for grain growth in nanocrystalline yttriastabilized zirconia," Nano Letters, vol. 3, no. 3, pp. 397-401, 2003.

[23] X. Minghan, D. Shifeng, S. Li, and Z. Jinchao, "Preparation of ultrafine cobalt oxalate powder by the precipitation method," Journal of the Chinese Ceramic Society, vol. 36, no. 3, p. 367, 2008.

[24] S. Majumdar, I. G. Sharma, A. C. Bidaye, and A. K. Suri, "A study on isothermal kinetics of thermal decomposition of cobalt oxalate to cobalt," Thermochimica Acta, vol. 473, no. 1-2, pp. 4549, 2008.

[25] S. Kawanishi, K. Isonishi, and K. Okazaki, "Grain growth and its kinetics of nanophase niobium aluminide produced by mechanical alloying," JIM Materials Transactions, vol. 34, no. 1, pp. 49-53, 1993. 

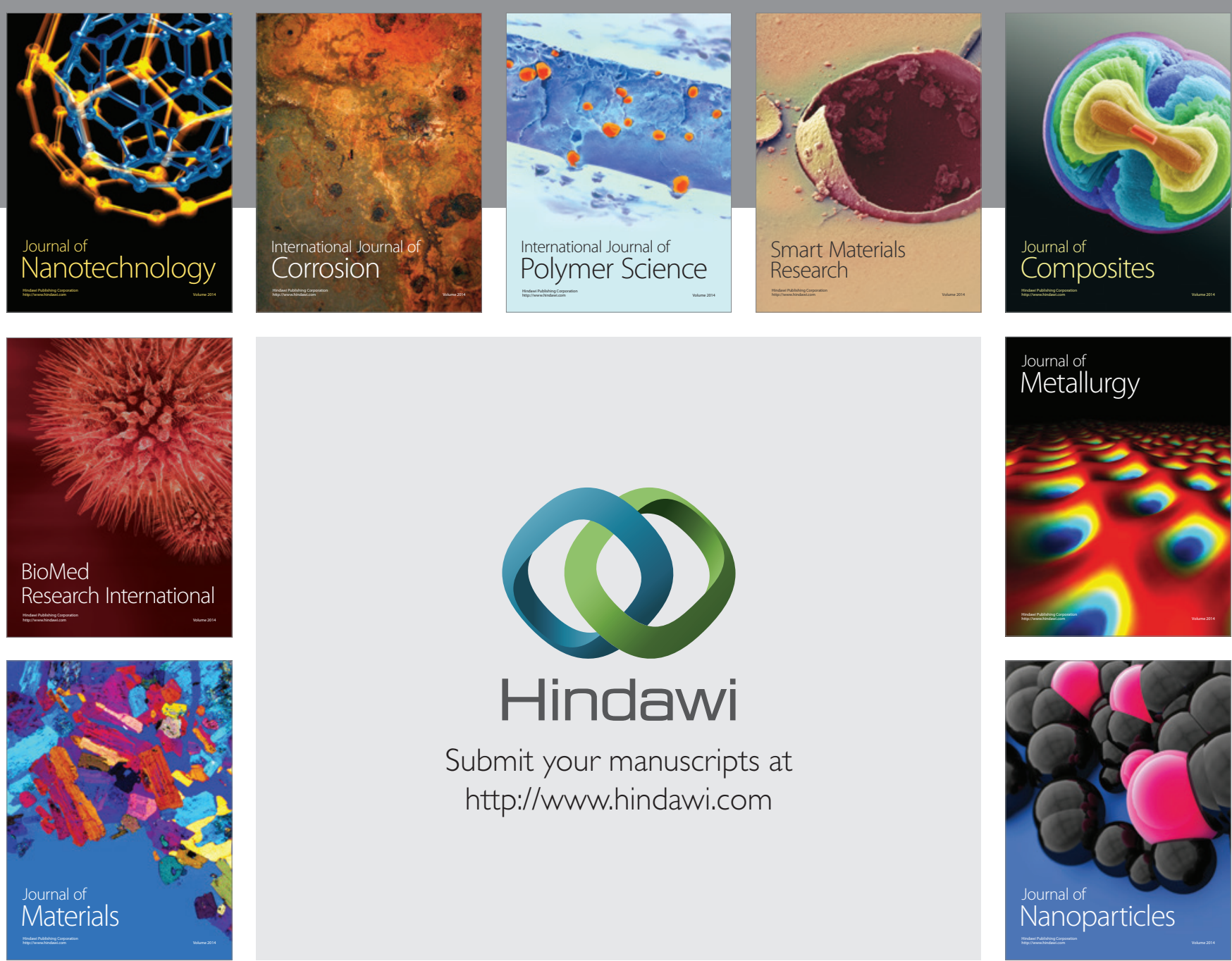

\section{Hindawi}

Submit your manuscripts at

http://www.hindawi.com

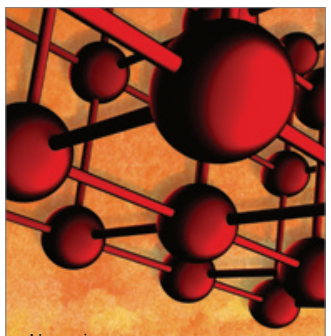

Materials Science and Engineering
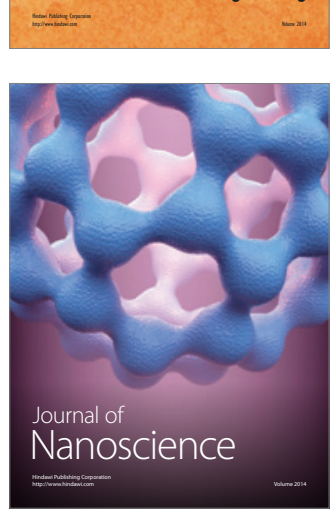
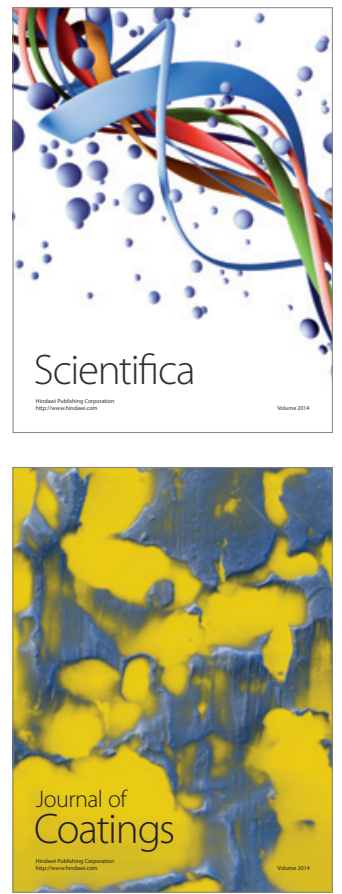
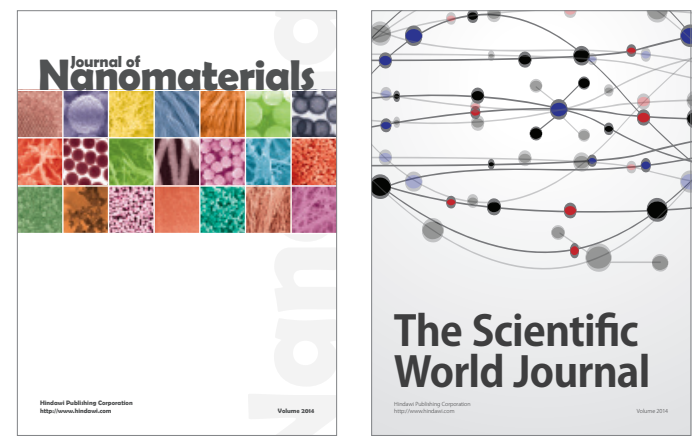

The Scientific World Journal
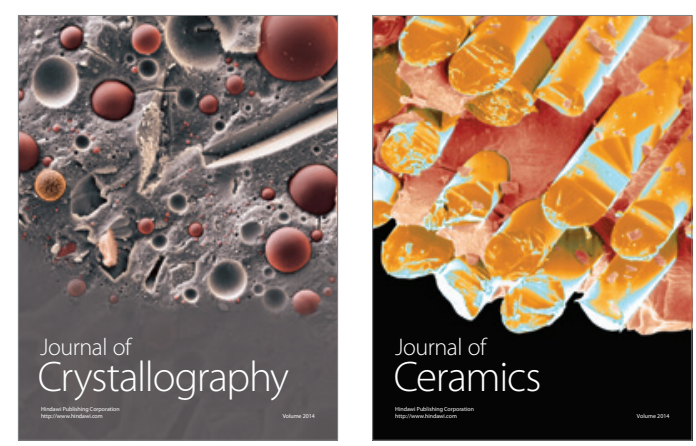
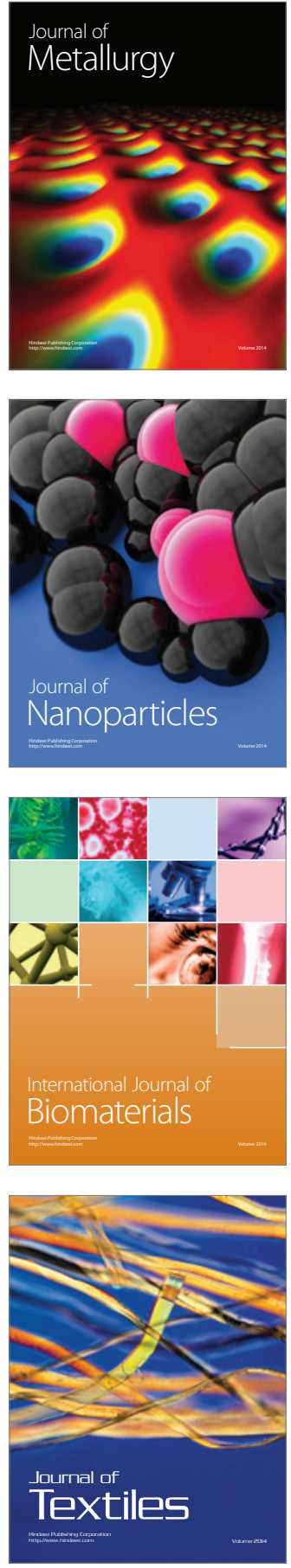